\title{
Glioma cordoide del III ventrículo. Nuevo caso y revisión de la literatura
}

M. Ortega-Martínez; J.M. Cabezudo; L.M. Bernal-García; I. Fernández-Portales; L. Gómez-Perals; R. Gómez de Tejada* y J.J. Pimentel*

Servicios de Neurocirugía y Anatomía Patológica*. Hospital Regional Universitario Infanta Cristina. Badajoz. España.

\section{Resumen}

El glioma cordoide del tercer ventrículo es una entidad tumoral muy infrecuente descrita por primera vez en 1998 por Brat y colaboradores; desde entonces se han descrito en la literatura tan sólo 39 casos. Presentamos un nuevo caso de glioma cordoide del tercer ventrículo en un paciente varón de 51 años, que fue tratado mediante resección quirúrgica completa, con buena evolución en el postoperatorio inmediato, pero que falleció súbitamente tres días después de la intervención por un probable embolismo pulmonar. Presentamos las características histológicas del tumor y realizamos una revisión de la literatura.

PALABRAS CLAVE: Glioma cordoide. Neoplasias cordoides. Tercer ventrículo. Tromboembolismo pulmonar.

\section{Summary}

Chordoid glioma of the third ventricle is an infrequent brain tumour that was described for the first time by Brat et al. in 1998; since then, only 39 cases have been reported. We present a new case of chordoid glioma of the third ventricle in a 51-year-old-man that was treated with total surgical removal, with a good initial postoperative evolution. Sudden death, most likely due to a massive pulmonary embolism, occurred in the third postoperative day. We present the histological characteristics of the tumour and review the literature regarding this entity.

KEY WORDS: Chordoid glioma. Chordoid neoplasms. Pulmonary embolism. Third ventricle.

\section{Introducción}

En 1998 Brat y cols. ${ }^{3}$ publicaron un trabajo en el que recogían una serie de ocho neoplasias localizadas en la

Recibido; 16-01-06. Aceptado: 16-02-06 parte anterior del III ventrículo, que presentaban un aspecto histológico característico de las neoplasias cordoides, pero con unas características inmunohistoquímicas muy peculiares, en especial por su positividad para la glioproteína fibrilar ácida (GFAP). En base a sus características histológicas, inmunohistoquímicas y ultraestructurales, junto a su específica localización, consideraron que se trataba de una nueva entidad tumoral que denominaron "glioma cordoide". Dos años después fue incluido en la clasificación de tumores de la Organización Mundial de la Salud entre los "Tumores neuroepiteliales de origen incierto", junto al astroblastoma y la gliomatosis cerebri. Desde ese primer trabajo hasta la actualidad se han publicado 39 casos de gliomas cordoides, siendo todavía un escaso número para poder sacar conclusiones definitivas en cuanto a su epidemiología, clínica, tratamiento y pronóstico. Presentamos un nuevo caso de glioma cordoide del III ventriculo y revisamos la literatura existente sobre estas neoplasias.

\section{Caso clínico}

Varón de 51 años, con antecedentes de DM tipo 2 y HTA, que ingresa en nuestro Hospital por un cuadro de pérdida de memoria y desorientación de un mes y medio de evolución, asociado desde hacía 20 días a ataxia e incontinencia urinaria. En la TC de cráneo realizada al ingreso se observó una hidrocefalia biventricular obstructiva producida por una tumoración localizada en el III ventrículo, que ocluía ambos forámenes de Monro, y que se trató mediante una derivación biventriculo-peritoneal urgente (Fig. lA). No se apreciaron alteraciones visuales ni endocrinas. La RM mostraba una tumoración ovoidea, de $3 \mathrm{~cm}$ de diámetro en el eje craneocaudal, $4 \mathrm{~cm}$ en el eje anteroposterior y $2.5 \mathrm{~cm}$ en el eje transverso, localizada en la parte anterior del III ventriculo, que se delimitaba fácilmente de los tejidos adyacentes y no presentaba edema periférico. La lesión era hiperdensa en la TC de cráneo e isointensa en la $\mathrm{RM}$, tanto en las secuencias ponderadas en $\mathrm{T} 1$ como en $\mathrm{T} 2$, y presentaba una ávida captación de contrastes yodado y paramagnético, con algunas zonas centrales hipocaptantes (Fig. 2). A los 13 días de la primera intervención se realizó 


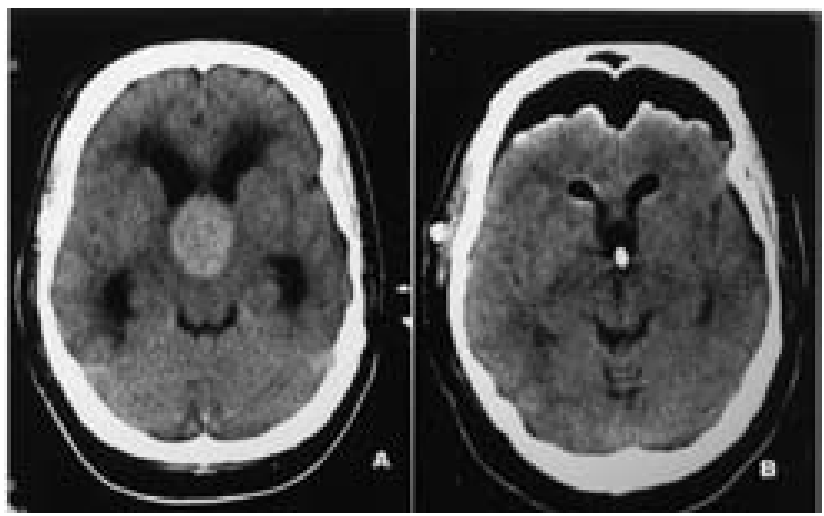

Figura 1. TC de cráneo pre y postquirúrgico, sin contraste A) Se aprecia una masa hiperdensa en relación con el parénquima cerebral, localizada en el tercer ventriculo, que produce una hidrocefalia obstructiva biventricular, por oclusión de ambos forámenes de Monro. B) TC de control postquirúrgica: descarta la presencia de complicaciones hemorrágicas, observándose la presencia de neumocéfalo y neumoventriculo; no se aprecia tumor residual (compárese con figura $I$ A)

un abordaje al III ventrículo por vía transcallosa. Durante la cirugía se observó una tumoración rojo-grisácea, poco sangrante y bien delimitada de las paredes ventriculares, de las que se disecaba sin dificultad, salvo en la zona inferior derecha, que se correspondía con el hipotálamo, donde se perdía el plano de clivaje. La extirpación fue macroscópicamente completa. Tras la intervención el paciente despertó sin déficits focales, obedecía órdenes, no presentó alteraciones endocrinas y sólo se apreciaba una bradipsiquia en probable relación con la vía de abordaje. La TC de control a las 12 horas de la intervención descartó la presencia de complicaciones hemorrágicas y sólo mostraba la presencia de neumocéfalo y neumoventrículo, bien tolerados por el paciente (Fig. 1B). A las 24 horas el paciente fue trasladado a planta donde se mantuvo estable hasta el tercer día en que, durante su movilización rutinaria, presentó parada cardiorrespiratoria con disociación electromecánica, intensa ingurgitación yugular y edema en esclavina, falleciendo a pesar de las medidas de resucitación cardiopulmonar; el cuadro clínico se atribuyó a un tromboembolismo pulmonar (TEP) masivo aunque no se pudo confirmar ante la falta de autorización familiar para estudio necrópsico.

\section{Anatomía patológica}

Al microscopio óptico y con tinción H/E se apreciaba una tumoración formada por células poligonales, con abundante citoplasma eosinófilo y núcleos de tamaño medio, redondeados y de cromatina granular fina. No se veían atipias y las mitosis eran excepcionales. En unas zonas for-

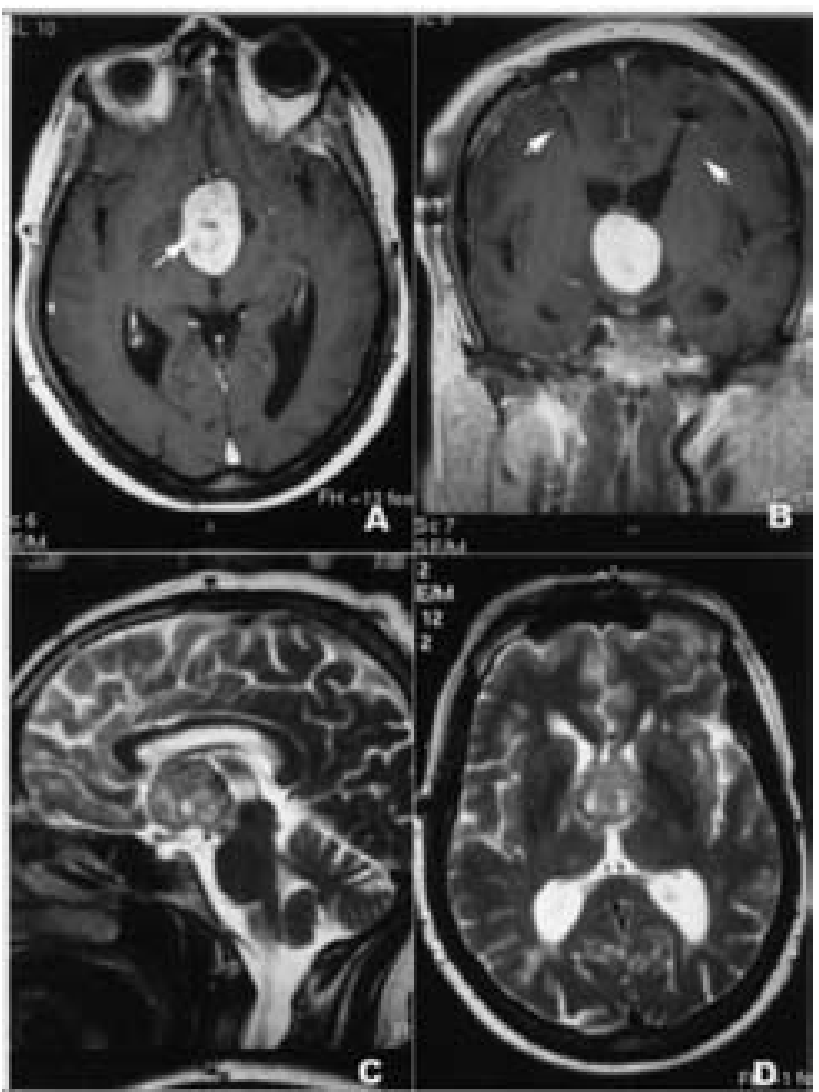

Figura 2: Aspecto del tumor en la RM. En los cortes superiores se presentan secuencias en T1 con contraste paramagnético. Se aprecian algunas zonas de hipocaptación intratumoral (A, flecha). En la imagen coronal (B) se aprecia el artefacto producido por los drenajes ventriculares (flechas). En los cortes inferiores se presentan cortes sagital y axial de RM ponderada en T2. Se observa la isointensidad del tumor con el parénquima cerebral y la ausencia de edema vasogénico.

maban masas sólidas y se acompañaban de un infiltrado linfoplasmocitario intersticial, con frecuentes cuerpos de Russell; en otras zonas, las células eran menos abundantes y se agrupaban en nidos y cordones en el seno de una matriz mixoide PAS positiva, bien evidente (Fig. 3). El estudio inmunohistoquímico (Fig. 4) mostró que las células tumorales expresaban de forma difusa GFAP, Vimentina y Citoqueratinas AE-1 y AE-3, así como focalmente EMA, proteína S-100 y CD-34. El índice Ki-67 fue menor del 2\%.

\section{Discusión}

Desde que Brat y cols. publicaran su trabajo sobre los gliomas cordoides del III ventriculo en el año $1998^{3}$, 40 de estos tumores, incluyendo el presente caso, han sido identificados y publicados en la literatura. (Tabla I). 


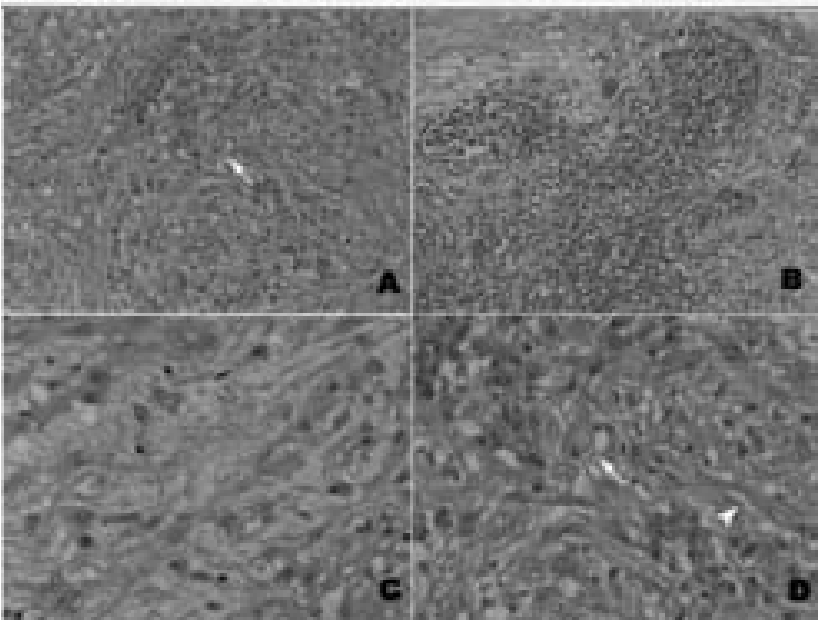

Figura 3. Aspecto histológico de la tumoración (hematoxilinaleosina). A) Nidos de células epiteloides y algunos cuerpos de Russell (flecha); B) Acúmulos linfoides; C) Areas mixoides; D) Cuerpos de Russell (flecha) y fibras de Rosenthal en la periferia (cabeza de flecha)

Del examen de los casos publicados se observa que se trata de una tumoración con predilección por el sexo femenino (relación mujer/varón de 1.7/1), que afecta de forma predominante pacientes entre los 30 y los 60 años, con una edad media de 45.7 años, si bien se han descrito casos en niños ${ }^{6} \mathrm{y}$ en pacientes ancianos ${ }^{3}$. (Gráfico I).

Se localizan en la parte anterior del III ventrículo, en estrecha relación con el hipotálamo y las estructuras de la región selar, lo que origina una gran riqueza sintomática, en forma de alteraciones visuales (defectos campimétricos, disminución de la agudeza visual), endocrinas (amenorrea, hipotiroidismo, diabetes insípida), alteraciones del comportamiento (psicosis, alteración de la memoria) o aumento de peso, y originando también síntomas debidos al aumento de

\section{$\begin{array}{cc}\text { N-DE } & \text { DISTRIBUCION DEL GLIOMA CORDOIDE } \\ \text { CASOS } & \text { POR EDAD Y SEXO }\end{array}$}

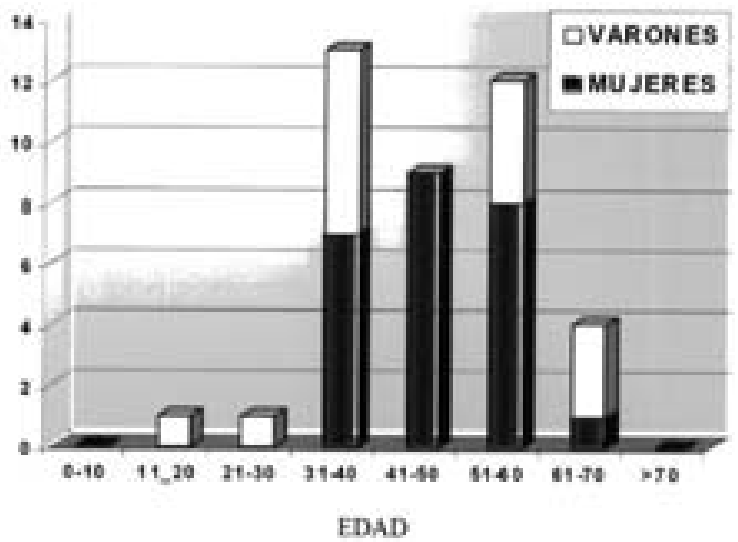

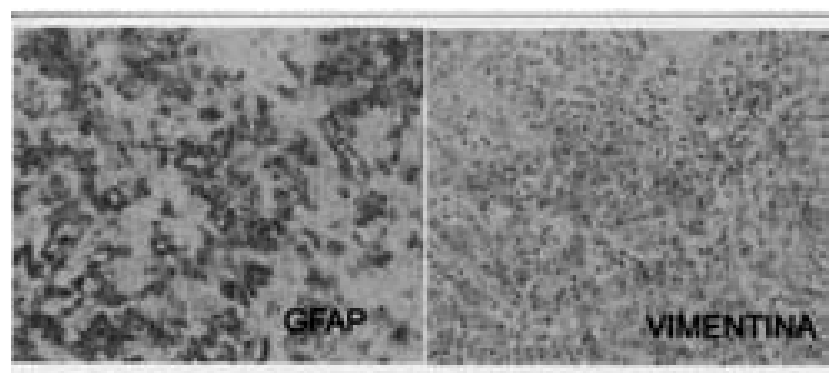
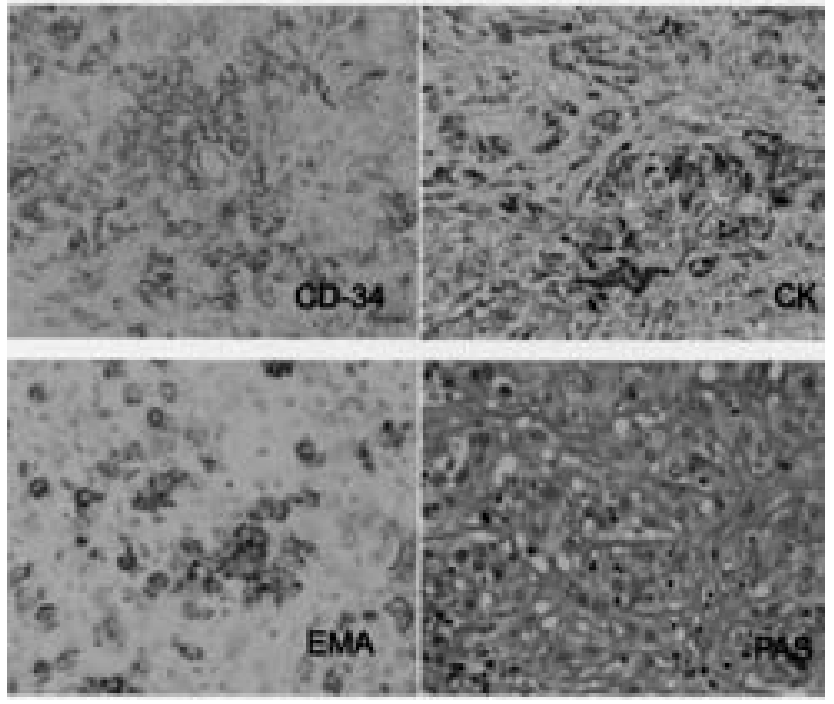

Figura 4. Inmunohistoquímica. Las células tumorales expresan de forma difusa GFAP, Vimentina y Citoqueratinas y de forma focal CD-34 y EMA. Tinción PAS positiva de la matriz mixoide.

la presión intracraneal o la hidrocefalia que pueden originar, incluyendo "dropp attacks" y pérdidas de conocimiento recidivantes de escasa duración ${ }^{3,12,19,27}$.

Desde el punto de vista de la neuroimagen, se trata de una lesión ovoidea, localizada en la región anterior del III ventrículo, hiperdensa en el TC e isointensa en la RM, tanto en las secuencias ponderadas en T1 como en T2. Capta contraste de forma ávida y uniforme, si bien en ocasiones presenta algunas zonas centrales de hipocaptación, que pudieran corresponder a quistes o necrosis, si bien en el estudio histológico no se ha apreciado la presencia de dicha necrosis $^{18}$. En ocasiones presenta edema perilesional, que suele ser bilateral y simétrico y se desplaza por los tractos ópticos. En todos los casos el tumor está separado de la hipófisis. Su eje mayor es craneocaudal y suele desplazar el infundíbulo hacia atrás, al contrario que los quistes de la bolsa de Rathke y los hamartomas hipotalámicos, que lo desplazan hacia delante ${ }^{18}$.

Tanto la histología como la inmunohistoquímica son muy características y uniformes en estos tumores. Presentan cordones o nidos de células epiteloides, con abundante 
Tabla I

Resúmen de los 40 casos publicados de glioma cordoide

\begin{tabular}{|c|c|c|c|c|c|c|}
\hline $\mathbf{N}^{\circ}$ & Autor & Edad/Sexo & Clínica & Tratamiento & $\begin{array}{l}\text { Complicaciones } \\
\text { postquirúrgicas }\end{array}$ & $\begin{array}{l}\text { Resultados y periodo de } \\
\text { seguimiento }\end{array}$ \\
\hline 1 & $\begin{array}{l}\text { Brat y cols, } \\
1998^{3}\end{array}$ & $50 / \mathrm{M}$ & $\begin{array}{l}\text { Letargia, } \\
\text { incontinencia } \\
\text { urinaria, ataxia }\end{array}$ & Qx total & NC & NC \\
\hline 2 & $\begin{array}{l}\text { Brat y cols, } \\
1998^{3}\end{array}$ & $70 / \mathrm{V}$ & Ataxia & Qx subtotal & NC & Sin recidiva (1 a) \\
\hline 3 & $\begin{array}{l}\text { Brat y cols, } \\
1998^{3}\end{array}$ & 59 / M & $\begin{array}{l}\text { Hidrocefalia } \\
\text { obstructiva }\end{array}$ & $\begin{array}{l}\text { Qx subtotal + } \\
\text { RT }\end{array}$ & $\mathrm{NC}$ & $\begin{array}{l}\text { Recidiva y EXITUS por la } \\
\text { enfermedad ( } 3 \text { a) } \\
\text { (Autopsia: gran recidiva tumoral) }\end{array}$ \\
\hline 4 & $\begin{array}{l}\text { Brat y cols, } \\
1998^{3}\end{array}$ & 47 / M & $\begin{array}{l}\text { Hidrocefalia } \\
\text { obstructiva }\end{array}$ & Qx subtotal & NC & $\begin{array}{l}\text { Recidiva ( } 5 \text { my éxitus (8m) } \\
\text { por complicaciones médicas. } \\
\text { No consta autopsia }\end{array}$ \\
\hline 5 & $\begin{array}{l}\text { Brat y cols, } \\
1998^{3}\end{array}$ & $\begin{array}{l}31 \text { / M } \\
\text { vómitos, }\end{array}$ & $\begin{array}{l}\text { Cefalea, nausea, } \\
\text { incopes? }\end{array}$ & Qx total & NC & Sin recidiva (6 m) \\
\hline 6 & $\begin{array}{l}\text { Brat y cols, } \\
1998^{3}\end{array}$ & $56 / \mathrm{M}$ & $\begin{array}{l}\text { Hipotiroidismo, } \\
\text { diabetes insípida }\end{array}$ & Qx subtotal & $\begin{array}{l}\text { Disfunción } \\
\text { hipotalámica y } \\
\text { alteración de memoria }\end{array}$ & Sin recidiva (1 a) \\
\hline 7 & $\begin{array}{l}\text { Brat y cols, } \\
1998^{3}\end{array}$ & $31 / \mathrm{M}$ & $\begin{array}{l}\text { Hipotiroidismo, } \\
\text { aumento de peso }\end{array}$ & Qx subtotal + RT & $\begin{array}{l}\text { Alteración } \\
\text { campimétrica }\end{array}$ & $\begin{array}{l}\text { Lento crecimiento del } \\
\text { tumor residual }(4 \mathrm{a})\end{array}$ \\
\hline 8 & $\begin{array}{l}\text { Brat y cols, } \\
1998^{3}\end{array}$ & 35 / M & Amenorrea, psicosis & Qx subtotal & $\mathrm{NC}$ & $\begin{array}{l}\text { EXITUS postquirúrgico } \\
\text { por TEP. No consta } \\
\text { autopsia }\end{array}$ \\
\hline 9 & $\begin{array}{l}\text { Reifenberger y } \\
\text { cols, } 1999^{20}\end{array}$ & $56 / \mathrm{M}$ & $\begin{array}{l}\text { Cefalea, fatiga, } \\
\text { alteraciones } \\
\text { visuales }\end{array}$ & $\begin{array}{l}\text { Qx subtotal + } \\
\text { GK }\end{array}$ & $\begin{array}{l}\text { Alteración en la } \\
\text { memoria y } \\
\text { somnolencia } \\
\text { postquirúrgica, } \\
\text { transitoria }\end{array}$ & Sin recidiva (3.5 a) \\
\hline 10 & $\begin{array}{l}\text { Reifenberger y } \\
\text { cols, } 1999^{20}\end{array}$ & 53 / M & $\begin{array}{l}\text { Alteraciones } \\
\text { visuales, aumento } \\
\text { de peso }\end{array}$ & Qx total ¿? & Sin complicaciones & $\mathrm{NC}$ \\
\hline 11 & $\begin{array}{l}\text { Reifenberger y } \\
\text { cols, } 1999^{20}\end{array}$ & $65 / V$ & $\begin{array}{l}\text { Alteraciones } \\
\text { lenguaje, parálisis } \\
\text { facial periférica }\end{array}$ & Qx total & Sin complicaciones & $\begin{array}{l}\text { EXITUS postquirúrgico } \\
\text { por TEP. No autopsia. } \\
\text { ANTECEDENTES TVP }\end{array}$ \\
\hline 12 & $\begin{array}{l}\text { Reifenberger y } \\
\text { cols, } 1999^{20}\end{array}$ & $35 / V$ & Psicosis & Qx total & $\mathrm{NC}$ & $\begin{array}{l}\text { EXITUS postquirúrgico } \\
\text { por TEP ( } 2 \text { semanas). } \\
\text { No autopsia }\end{array}$ \\
\hline 13 & $\begin{array}{l}\text { Vatjai y cols, } \\
1999^{28}\end{array}$ & $60 / \mathrm{M}$ & $\begin{array}{l}\text { Cefalea, pérdida de } \\
\text { memoria, } \\
\text { somnolencia }\end{array}$ & Qx subtotal & $\begin{array}{l}\text { Hiponatremia } \\
\text { transitoria }\end{array}$ & $\begin{array}{l}\text { EXITUS postquirúrgico } \\
\text { por complicaciones respiratorias } \\
\text { (10 días) (Autopsia: traqueobronquitis) }\end{array}$ \\
\hline 14 & $\begin{array}{l}\text { Tonami y cols, } \\
2000^{27}\end{array}$ & 42 / M & $\begin{array}{l}\text { Amenorrea, pérdida } \\
\text { de memoria, } \\
\text { sincopes? }\end{array}$ & $\begin{array}{l}\text { Qx subtotal + RT } \\
+ \text { RQx } \\
\text { estereotáctica }\end{array}$ & $\mathrm{NC}$ & Sin recidiva (9 m) \\
\hline 15 & $\begin{array}{l}\text { Ricoy y cols, } \\
2000^{21}\end{array}$ & $41 / \mathrm{M}$ & $\begin{array}{l}\text { Alteraciones } \\
\text { visuales }\end{array}$ & Qx total & $\begin{array}{l}\text { Sin complicaciones. } \\
\text { Mejoría AV }\end{array}$ & Sin recidiva (13 m) \\
\hline 16 & $\begin{array}{l}\text { Castellano- } \\
\text { Sanchez y cols, } \\
2000^{5}\end{array}$ & $36 / V$ & $\begin{array}{l}\text { Alteraciones } \\
\text { visuales }\end{array}$ & $\begin{array}{l}\text { Qx total (en } 2 \\
\text { pasos) }\end{array}$ & $\begin{array}{l}\text { Crisis postquirúrgicas } \\
\text { inmediatas }\end{array}$ & NC \\
\hline 17 & $\begin{array}{l}\text { Pomper y cols, } \\
2001^{18}\end{array}$ & 59 / V & Cefalea & Qx subtotal & NC & $\mathrm{NC}$ \\
\hline 18 & $\begin{array}{l}\text { Pomper y cols } \\
2001^{18}\end{array}$ & $41 / \mathrm{M}$ & Cefalea & Qx subtotal & $\mathrm{NC}$ & $\mathrm{NC}$ \\
\hline
\end{tabular}




\begin{tabular}{|c|c|c|c|c|c|c|}
\hline $\mathbf{N}^{\circ}$ & Autor & Edad/Sexo & Clínica & Tratamiento & $\begin{array}{l}\text { Complicaciones } \\
\text { postquirúrgicas }\end{array}$ & $\begin{array}{l}\text { Resultados y periodo de } \\
\text { seguimiento }\end{array}$ \\
\hline 19 & $\begin{array}{l}\text { Pomper y cols, } \\
2001^{18}\end{array}$ & $36 / V$ & $\begin{array}{l}\text { Pérdida de } \\
\text { memoria, } \\
\text { alteraciones } \\
\text { visuales }\end{array}$ & Biopsia & NC & NC \\
\hline 20 & $\begin{array}{l}\text { Pomper y cols, } \\
2001^{18}\end{array}$ & $40 / \mathrm{M}$ & $\begin{array}{l}\text { Cefalea, diplopia, } \\
\text { alteraciones } \\
\text { visuales }\end{array}$ & Qx subtotal & NC & NC \\
\hline 21 & $\begin{array}{l}\text { Hanbali y cols, } \\
2001^{11}\end{array}$ & $57 / \mathrm{V}$ & $\begin{array}{l}\text { Cefalea, pérdida de } \\
\text { olfato, anorexia }\end{array}$ & Qx subtotal + RT & $\begin{array}{l}\text { Ictus parietal } \\
\text { isquémico }\left(1^{\text {er }} \text { día }\right. \\
\text { postqx }) \text { e IAM ( }\left(2^{\circ} \text { días }\right. \\
\text { postqx). Hiponatremia } \\
\text { (a los } 10 \text { meses }) \\
\text { DI tras la 2' cirugía }\end{array}$ & $\begin{array}{l}\text { Recidiva }(11 \text { meses }) \rightarrow \text { Qx } \\
\text { total } \rightarrow \text { EXITUS } 2 \text { meses } \\
\text { postqx por IAM masivo } \\
\text { No consta autopsia }\end{array}$ \\
\hline 22 & $\begin{array}{l}\text { Cenacchi y } \\
\text { cols, } 2001^{7}\end{array}$ & $34 / \mathrm{V}$ & $\mathrm{NC}$ & Qx total & NC & Sin recidiva (2 a) \\
\hline 23 & $\begin{array}{l}\text { Cenacchi y } \\
\text { cols, } 2001^{7}\end{array}$ & $40 / \mathrm{V}$ & $\mathrm{NC}$ & Qx total & NC & Sin recidiva (3 a) \\
\hline 24 & $\begin{array}{l}\text { Cenacchi y } \\
\text { cols, } 2001^{7}\end{array}$ & $43 / \mathrm{M}$ & $\mathrm{NC}$ & Qx total & NC & $\begin{array}{l}\text { EXITUS postquirúrgico } \\
\text { por TEP ( } 15 \text { días) } \\
\text { Autopsia: TEP masivo }\end{array}$ \\
\hline 25 & $\begin{array}{l}\text { Galloway y } \\
\text { cols, } 2001^{9}\end{array}$ & $54 / \mathrm{V}$ & $\begin{array}{l}\text { Hemiparesia } \\
\text { ataxia, alteraciones } \\
\text { visuales }\end{array}$ & Qx total & Sin complicaciones & Sin recidiva (9 m) \\
\hline 26 & $\begin{array}{l}\text { Castellano- } \\
\text { Sánchez y cols, } \\
2001^{6}\end{array}$ & $12 / \mathrm{V}$ & $\begin{array}{l}\text { Alteraciones } \\
\text { visuales }\end{array}$ & Qx subtotal & NC & $\begin{array}{l}\text { NC } \\
\text { Metaplasia cartilaginosa }\end{array}$ \\
\hline 27 & $\begin{array}{l}\text { Oda y cols, } \\
2002^{16}\end{array}$ & $25 / \mathrm{V}$ & $\begin{array}{l}\text { Aumento apetito, } \\
\text { alteraciones } \\
\text { memoria }\end{array}$ & Qx total & $\begin{array}{l}\text { DI, hipertermia, } \\
\text { hiponatremia y } \\
\text { alteración en la } \\
\text { memoria, transitorias }\end{array}$ & Sin recidiva (17 m) \\
\hline 28 & $\begin{array}{l}\text { Pasquier y cols, } \\
2002^{17}\end{array}$ & $35 / V$ & $\begin{array}{l}\text { Cefalea, nauseas, } \\
\text { alteraciones } \\
\text { visuales, insomnio }\end{array}$ & Qx subtotal & & Sin recidiva (68 m) \\
\hline 29 & $\begin{array}{l}\text { Pasquier y cols, } \\
2002^{17}\end{array}$ & 39 / M & $\begin{array}{l}\text { Cefalea, diplopia, } \\
\text { alteraciones } \\
\text { visuales }\end{array}$ & Qx subtotal & $\begin{array}{l}\text { Alteración en la } \\
\text { memoria, transitoria, y } \\
\text { aumento de peso ( } 20 \mathrm{Kg})\end{array}$ & Sin recidiva (16 m) \\
\hline 30 & $\begin{array}{l}\text { Grand y cols, } \\
2002^{10}\end{array}$ & $41 / \mathrm{M}$ & $\begin{array}{l}\text { Cefalea, } \\
\text { alteraciones } \\
\text { visuales }\end{array}$ & Qx subtotal & $\begin{array}{l}\text { DI, aumento de peso y } \\
\text { alteración en la } \\
\text { memoria. }\end{array}$ & NC \\
\hline 31 & $\begin{array}{l}\text { Taraszewska y } \\
\text { cols, } 2003^{26}\end{array}$ & $62 / V$ & Cefalea, DI & Qx total & $\begin{array}{l}\text { Disfunción ventilatoria } \\
\text { y coma, transitorio DI } \\
\text { y alteraciones visuales } \\
\text { IAM día } 14\end{array}$ & $\begin{array}{l}\text { EXITUS a las } 6 \text { semanas } \\
\text { por afectación cardiaca }\end{array}$ \\
\hline 32 & $\begin{array}{l}\text { Taraszewska y } \\
\text { cols, } 2003^{26}\end{array}$ & $51 / \mathrm{M}$ & $\begin{array}{l}\text { Hipersomnia y } \\
\text { alteraciones } \\
\text { visuales }\end{array}$ & Qx total & Sin complicaciones & Tumor residual (4 meses) \\
\hline 33 & $\begin{array}{l}\text { Raizer y cols, } \\
2003^{19}\end{array}$ & $57 / M$ & Sincopes & Qx total & $\begin{array}{l}\text { DI, FA transitoria, } \\
\text { meningitis postqx }\end{array}$ & Sin recidiva $(15 \mathrm{~m})$ \\
\hline 34 & $\begin{array}{l}\text { Nakajima y } \\
\text { cols, } 2003^{15}\end{array}$ & 49 / M & Cefalea & $\begin{array}{l}\text { Qx subtotal + } \\
\text { GK }\end{array}$ & $\begin{array}{l}\text { Alteración } \\
\text { concentración de Na, } \\
\text { transitoria. TEP } 1 \text { mes } \\
\text { postqx }\end{array}$ & Sin recidiva (2 a) \\
\hline 35 & $\begin{array}{l}\text { Sato y cols, } \\
2003^{22}\end{array}$ & $65 / \mathrm{M}$ & Cefalea & Qx subtotal & Sin complicaciones & $\begin{array}{l}\text { Sin recidiva ( } 2 \text { a) } \\
\text { EXITUS a los } 2.5 \text { a por } \\
\text { neumonía (no autopsia) }\end{array}$ \\
\hline
\end{tabular}




\begin{tabular}{|c|c|c|c|c|c|c|}
\hline $\mathbf{N}^{\circ}$ & Autor & Edad/Sexo & Clínica & Tratamiento & $\begin{array}{l}\text { Complicaciones } \\
\text { postquirúrgicas }\end{array}$ & $\begin{array}{l}\text { Resultados y periodo de } \\
\text { seguimiento }\end{array}$ \\
\hline 36 & $\begin{array}{l}\text { Suh y cols, } \\
2003^{25}\end{array}$ & $48 / \mathrm{M}$ & $\begin{array}{l}\text { Cefalea, mareos, } \\
\text { alteraciones } \\
\text { visuales }\end{array}$ & Qx total & $\begin{array}{l}\text { DI. } \\
\text { Mejoría campimétrica }\end{array}$ & $\begin{array}{l}\text { Sin recidiva }(17 \mathrm{~m}) \\
\text { ASOCIABA QUISTE DE } \\
\text { RATHKE }\end{array}$ \\
\hline 37 & $\begin{array}{l}\text { Bucolliero y } \\
\text { cols, 2004 }\end{array}$ & $56 / M$ & Hallazgo & Qx total & Sin complicaciones & Sin recidiva (8 $\mathrm{m})$ \\
\hline 38 & $\begin{array}{l}\text { Kurian y cols, } \\
2005^{12}\end{array}$ & $32 / \mathrm{M}$ & $\begin{array}{l}\text { Alteraciones } \\
\text { visuales, aumento } \\
\text { de peso, amenorrea }\end{array}$ & $\begin{array}{l}\text { Qx subtotal + } \\
\text { radioterapia } \\
\text { intersticial }\end{array}$ & $\begin{array}{l}\text { Disfunción } \\
\text { hipotalámica } \\
\text { persistente }\end{array}$ & $\begin{array}{l}15 \text { m. Mal estado clínico } \\
\text { Sin recidiva ¿? }\end{array}$ \\
\hline 39 & $\begin{array}{l}\text { Kurian y cols, } \\
2005^{12}\end{array}$ & $37 / \mathrm{M}$ & $\begin{array}{l}\text { Cefalea, pérdida de } \\
\text { memoria, síncopes, } \\
\text { hidrocefalia aguda }\end{array}$ & $\begin{array}{l}\text { Biopsia } \\
\text { estereotáctica+ } \\
\text { radioterapia } \\
\text { intersticial }\end{array}$ & $\begin{array}{l}\text { DI hipotermia, } \\
\text { hipotension }\end{array}$ & $\begin{array}{l}\text { Edema postRT y recidiva } \\
\text { ( } 9 \text { meses) } \rightarrow \text { Qx subtotal } \\
\rightarrow \text { EXITUS postquirúrgico } \\
\text { (2 semanas) (Autopsia: } \\
\text { Sepsis) }\end{array}$ \\
\hline 40 & $\begin{array}{l}\text { Ortega y cols, } \\
2006 \text { (presente } \\
\text { caso) }\end{array}$ & $51 / \mathrm{V}$ & $\begin{array}{l}\text { Hidrocefalia } \\
\text { obstructiva }\end{array}$ & Qx total & Bradipsiquia & $\begin{array}{l}\text { EXITUS postquirúrgico } \\
\text { por TEP ( } 3 \text { días, no } \\
\text { autopsia) }\end{array}$ \\
\hline
\end{tabular}

AV: agudeza visual. DI: Diabetes insípida. FA: Fibrilación auricular. GK: Gamma-knife. IAM: Infarto agudo de miocardio. M: Mujer. NC: No consta. Qx: Cirugía. RQx: Radiocirugía. RT: Radioterapia. TEP: Tromboembolismo pulmonar. V: Varón.

citoplasma eosinófilo en una matriz mucinosa PAS positiva, sin atipias, pleomorfismo, necrosis o proliferación vascular. Las mitosis son muy raras o ausentes. Hay un infiltrado linfoplasmocitario prominente. En el parénquima adyacente es frecuente encontrar células gliales reactivas, fibras de Rosenthal y un infiltrado crónico inflamatorio. Las células tumorales siempre expresan de forma difusa GFAP y Vimentina, y en la mayoría de los casos presentan una fuerte coexpresión de carácter focal para Citoqueratinas y CD 34, siendo más débil y también focal la reactividad para la proteína $\mathrm{S} 100$ y $\mathrm{EMA}^{3,4,7,17,21}$. La histología y el perfil inmunohistoquímico ayudan al diagnóstico diferencial, en el que destaca la diferenciación con otras neoplasias cordoides, concretamente el meningioma cordoide (negativo para GFAP y positivo para EMA) y el cordoma (gran presencia de células fisalíforas e intensamente positivo para citoqueratinas). Al microscopio electrónico se ven frecuentemente abundantes filamentos intermedios, complejos de unión bien desarrollados, membrana basal focal y proyecciones intercelulares que recuerdan microvellosidades ${ }^{6,17,19}$; estas características ultraestructurales, unidas al perfil inmunohistoquímico y a los hallazgos de genética molecular (concretamente, ausencia de alteraciones moleculares propias de los astrocitomas difusos del adulto y de los meningiomas), hace considerar a estos tumores como neoplasias originadas de células de estirpe ependimaria ${ }^{6,17,19,21}$, sugiriendo algunos su origen tanicítico ${ }^{22}$, mientras otros apuntan que su origen pudiera encontrarse en células ependimarias modificadas del órgano subcomisural ${ }^{7}$.

El tratamiento más adecuado de estos tumores, especialmente en lo que respecta a la necesidad de tratamiento complementario con RT o QT, está por determinarse. De los 40 casos descritos en la literatura, 38 fueron abordados quirúrgicamente, realizándose una resección completa en 18 de los pacientes y subtotal en 20. En los dos casos restantes se realizó biopsia de la lesión, uno de los cuales se completó con RT intersticial ${ }^{12}$, presentando una progresión tumoral masiva en 9 meses.

De los 18 pacientes con resección completa disponemos de seguimiento en 10 de ellos, dado que 5 fallecieron en el postoperatorio inmediato y en 3 no consta la evolución. En estos pacientes sólo hubo una recidiva ${ }^{26}$, si bien el tiempo de seguimiento en este grupo es escaso (14.9 meses de media, con un rango entre 4 y 36 meses). (Tabla II)

De los 20 pacientes con resección subtotal disponemos de seguimiento en 13 de ellos (2 fueron éxitus postquirúrgicos inmediatos y en 5 no consta evolución). En estos 13 casos se aplicó tratamiento complementario con diversas modalidades de radioterapia a 7 pacientes (ver Tabla II). Se ha observado recidiva o progresión tumoral en 4 de estos 13 casos (30\%), 3 de los cuales habían recibido RT fraccionada postquirúrgica; el tiempo de seguimiento fue de 25 meses de media, con rango entre 5 y 68 meses. En ninguno de los casos consta tratamiento quimioterápico.

Hemos de señalar que, además de las complicaciones propias de la cirugía de las lesiones del tercer ventriculo, la revisión de la literatura muestra una alta frecuencia de complicaciones tromboembólicas severas en estos pacientes (ictus isquémico, infarto agudo de miocardio, TEP), registrándose estas complicaciones en 8 pacientes incluido el nuestro, con un resultado de cinco muertes en el postoperatorio inmediato por dicha causa (ver Tabla I) y otras dos a las seis y ocho semanas de la cirugía respectivamente ${ }^{11,25}$. Si el riesgo de embolismo pulmonar sintomático en pacientes operados de un tumor cerebral está entre un 1.1 y un $5 \%^{1,2,8,13,24}$, en el caso del glioma cordoide se han 


\begin{tabular}{|c|c|c|c|c|}
\hline \multicolumn{5}{|c|}{$\begin{array}{l}\text { Tabla II } \\
\text { Extensión de la resección y tratamiento adicional } \\
\text { Los números entre paréntesis indican los casos en los que consta recidiva o progresión tumoral }\end{array}$} \\
\hline & $\begin{array}{l}\text { RESECCION } \\
\text { QUIRURGICA } \\
\text { COMPLETA }\end{array}$ & $\begin{array}{l}\text { RESECCION } \\
\text { QUIRURGICA } \\
\text { SUBTOTAL }\end{array}$ & BIOPSIA & TOTAL \\
\hline Pacientes sin seguimiento & 3 & 5 & 1 & 9 \\
\hline Exitus postquirúrgicos inmediatos & 5 & 2 & 0 & 7 \\
\hline No tto adicional & $10(1)$ & $6(1)$ & 0 & 16 \\
\hline Radioterapia fraccionada & 0 & $3(3)$ & 0 & 3 \\
\hline RT +0101 Radiocirugía con acelerador & 0 & 1 & 0 & 1 \\
\hline Gamma-Knife & 0 & 2 & 0 & 2 \\
\hline RT intersticial & 0 & 1 & $1(1)$ & 2 \\
\hline Quimioterapia & 0 & 0 & 0 & 0 \\
\hline TOTAL & 18 & 20 & 2 & 40 \\
\hline
\end{tabular}

sospechado embolismos pulmonares al menos en el 15\% de los pacientes, aunque los datos no se han confirmado en la mayoría de los casos con autopsia. Si bien es cierto que en estos pacientes se dan condiciones para el aumento de fenómenos trombóticos (tratamiento esteroideo, reposo en cama prolongado, posible déficit motor focal, localización tumoral en región supratentorial/supraselar, uso de vías centrales para administración de fármacos, largo tiempo quirúrgico) ${ }^{8}$, estas condiciones se comparten en su mayoría con otros tumores cerebrales. Parece que en estas neoplasias se produce un estado de hipercoagulabilidad humoral tras la cirugía que es mayor que en otros tumores. Morozov fue el primero en sugerir que factores humorales secretados por el tumor o por tejido cerebral normal puede ser responsable de la hipercoagulabilidad que se observa en los pacientes con tumores cerebrales ${ }^{14,29}$. Sawaya ha demostrado que la presencia de trombosis venosas en pacientes intervenidos por tumores cerebrales se correlaciona con alteraciones preoperatorias en la expresión de distintos componentes de la cascada de la coagulación y de la fibrinolisis; ha observando también que dichos parámetros hemostáticos difieren en relación con diferentes tipos de tumores, lo que sugiere que existen factores biológicos específicos de cada tumor ${ }^{23}$. En este sentido cabe pensar que exista algún factor espe- cífico de los gliomas cordoides que desencadenara tras la cirugía un mayor estado de hipercoagulabilidad sistémica del esperado. Por ello, consideramos adecuado iniciar una profilaxis intensa de los fenómenos tromboembólicos en los pacientes en los que se sospeche esta patología, con el uso de heparinas de bajo peso molecular a dosis adecuadas para pacientes de alto riesgo, asociados a sistemas de compresión neumática y movilización precoz.

\section{Conclusiones}

Los gliomas cordoides son neoplasias raras, de bajo grado histológico, pero cuya localización condiciona un alto riesgo quirúrgico. La resección completa, por el momento, parece el tratamiento de elección. En cuanto a las complicaciones postquirúrgicas, destaca una alta frecuencia de fenómenos tromboembólicos severos, por lo que se aconseja extremar las precauciones en estos pacientes.

\section{Bibliografía}

1. Agnelli, G., Piovella, F., Buoncristiani, P., Severi, P., Pini, M., D'Angelo, A., et al.: Enoxaparin plus compression stockings alone in the prevention of venous thromboemboslism after elec- 
tive neurosurgery. New Engl J Med. 1998; 339: 80-86.

2. Black, P.M., Baker, M.F., Snook, C.P.: Experience with external pneumatic calf compression in neurology and neurosurgery. Neurosurgery 1986; 18: 440-444.

3. Brat, D.J., Scheithauer, B.W., Staugatis, S.M., Cortez, S.C., Brecher, K., Burger, P.C.: Third ventricular chordoid glioma: a distinct clinicopathologic entity. J Neuropathol Exp Neurol. 1998; 57: 283-290.

4. Buccoliero, A.M., Caldarella, A., Gallina, P., Di Lorenzo, N., Taddei, A., Taddei, G.L.: Chordoid glioma: clinicopathologic profile and differential diagnosis of an uncommon tumor. Arch Pathol Lab Med. 2004; 128: e141-e145.

5. Castellano-Sánchez, A.A., Recine, M.A., Restrepo, R., Howard, L.H., Robinson, M.J.: Chordoid glioma: a novel tumor of the third ventricle. Ann Diagn Pathol. 2000; 4: 373-378.

6. Castellano-Sánchez, A.A., Schemankewitz, E., Mazewski, C., Brat, D.J.: Pediatric chordoid glioma with chondroid metaplasia. Pediatr Dev Pathol. 2001; 4: 564-567.

7. Cenacchi, G., Roncaroli, F., Cerasoli, S., Ficarra, G., Merli. G.A., Giangaspero, F.: Chordoid glioma of the third ventricle: an ultrastructural study of three cases with a histogenetic hypothesis. Am J Surg Pathol. 2001; 25: 401-405.

8. Danish, S.H., Burnett, M.G., Stein, S.C.: Prophylaxis for deep venous thrombosis in patients with craniotomies: a review. Neurosurg focus 2004; 17 (4): E2.

9. Galloway, M., Afshar, F., Geddes, J.F.: Chordoid glioma: an uncommon tumour of the third ventricle. $\mathrm{Br} \mathrm{J}$ Neurosurg. 2001; 15: 147-150.

10. Grand, S., Pasquier, B., Gay, E., Kremer, S., Remy, C., Le Bas, J.F.: Chordoid glioma of the third ventricle: CT and MRI, including perfusion data. Neuroradiology. 2002; 44: 842-846.

11. Hanbali, F., Fuller, G.N., Leeds, N.E., Sawaya, R.: Choroid plexus cyst and chordoid glioma. Report of two cases. Neurosurg focus 2001; 10 (6), article 5.

12. Kurian, K.M., Summers, D.M., Statham, P.F.X., Smith, C., Bell, J.E., Irnside, J.W.: Third ventricular chordoid glioma: clinicopathological study of two cases with evidence of a poor clinical outcome despite low grade histological features. Neuropathol Appl Neurobiol. 2005; 31: 354-361.

13. Missori, P., Lunardi, P., Salvati, M., Esposito, V., Oppido, P.: Pulmonary embolism in neurosurgical patients. Neurochirurgia 1991; 34 : 170-173.

14. Morozov, V.V.: The coagulant and anticoagulant blood systems in brain tumors of supratentorial localization. Zh Nevropatol Psikhiatr Im SS Korsakova 1968; 68: 505-509.

15. Nakajima, M., Nakasu, S., Hatsuda, N., Takeichi, Y., Watanabe, K., Matsuda, M.: Third ventricular chordoid glioma: case report and review of the literature. Surg Neurol. 2003; 59: 424-428.

16. Oda, M., Sasajima, T., Kinouchi, H., Sageshima, M., Mizoi, K.: Third ventricular chordoid glioma: report of a surgical case. No Shinkei Geka. 2002; 30: 973-979.

17. Pasquier, B., Péoc'h, M., Morrison, A.L., et al.: Chor- doid glioma of the third ventricle: a report of two new cases, with further evidence supporting an ependymal differentiation, and review of the literature. Am J Surg Pathol. 2002; 26: 1330-1342.

18. Pomper, M.G., Passe, T.J., Burger, P.C., Scheithauer, B.W., Brat, D.J.: Chordoid glioma: a neoplasm unique to the hypothalamus and anterior third ventricle. AJNR 2001; 22:464-469.

19. Raizer, J.J., Shetty, T., Gutin, P.H., et al.: Chordoid glioma: report of a case with unusual histologic features, ultrastructural study and review of the literature. J Neurooncol. 2003; 63: 39-47.

20. Reifenberger, G., Weber, T., Weber, R.G., et al.: Chordoid glioma of the third ventricle: immunohistochemical and molecular genetic characterization of a novel tumor entity. Brain Pathol. 1999; 9: 617-626.

21. Ricoy, J.R., Lobato, R.D., Baez, B., Cabello, A., Martínez, M.A., Rodríguez, G.: Suprasellar chordoid glioma. Acta Neuropathol (Berl). 2000: 99: 699-703.

22. Sato, K., Kubota, T., Ishida, M., Yoshida, K., Takeuchi, H., Handa, Y.: Immunohistochemical and ultrastructural study of chordoid glioma of the third ventricle: its tanycytic differentiation. Acta Neuropathol (Berl). 2003; 106: 176-180.

23. Sawaya, R., Glas-Greenwalt, P.: Postoperative venous thromboembolism and brain tumours: Part II. Hemostatic profile. J Neurooncol 1992; 14: 127-134.

24. Smith, S.F., Sympson, J.M., Sekhon, L.H.S.: Prophylaxis for deep venous thrombosis in neurosurgical oncology: review of 2779 admissions over a 9-year period. Neurosurg focus 2004; 17 (4): E4.

25. Suh, Y.L., Kim, N.R., Kim, J.H., Park, S.H.: Suprasellar chordoid glioma combined with Rathke's cleft cyst. Pathol Int. 2003; 53: 780-785.

26. Taraszewska, A., Bogucki, J., Andrychowski, J., Koszewski, W., Czernicki, Z.: Clinicopathological and ultrastructural study in two cases of chordoid glioma. Folia Neuropathol. 2003; 41:175-182.

27. Tonami, H., Kamehiro, M., Oguchi, M., et al.: Chordoid glioma of the third ventricle: CT and MR findings. J Comput Assist Tomogr. 2000; 24: 336-338.

28. Vajtai, I., Varga, Z., Scheithauer, B.W., Bodosi, M.: Chordoid glioma of the third ventricle: confirmatory report of a new entity. Hum Pathol. 1999; 30: 723-726.

29. Walsh, D.C., Kakkar, A.K.: Thromboembolism in brain tumors. Curr Opin Pulm Med 2001; 7: 326-331.

Ortega-Martínez, M.; Cabezudo, J.M.; Bernal-García, L.M.; Fernández-Portales, I.; Gómez-Perals, L.; Gómez de Tejada, R.; Pimentel, J.J.: Glioma cordoide del III ventriculo. Nuevo caso y revisión de la literatura. Neurocirugía 2007; 18: 115-122.

Correspondencia postal: Dra. M. Ortega Martínez. Servicio de Neurocirugía. Hospital Regional Universitario Infanta Cristina. Avda de Elvas s/n. 06006 Badajoz. 